\title{
Estrategias metodológicas para el estudio de la inserción sociolaboral de las personas con discapacidad
}

\author{
Methodological strategies for the study of \\ social and professional integration of people \\ with disabilities
}

\section{Resumen}

El interés de las ciencias sociales por la discapacidad es relativamente reciente. En la última década se ha generado un aumento de la producción científica que ha contribuido al conocimiento de la propia discapacidad. En aras de mejorar la labor investigadora en materia de discapacidad, el presente trabajo centra su objetivo en el análisis de las metodologías de investigación social más utilizadas para el estudio de los procesos de inserción sociolaboral de la población con discapacidad en España. La metodología seguida ha consistido en la revisión de los estudios existentes en España sobre discapacidad, analizando las características de las técnicas empleadas (cuantitativas y cualitativas): así como los diferentes marcos teóricos utilizados (conceptos de discapacidad y exclusión social). Las principales conclusiones indican un predominio de análisis cualitativos y metodologías mixtas, un enfoque multidimensional y un modelo biopsicosocial de la discapacidad con una leve tendencia hacia una plena ciudadanía.

\section{Palabras clave}

Discapacidad, metodología de investigación social, diversidad funcional, integración sociolaboral, fuentes de datos.

\section{Abstract}

The interest of the social sciences in disability is relatively recent. In the last decade the scientific production has increased and contributed to the knowledge of the disability itself. In order to improve the research work on disability, this paper focuses its objective on the analysis of the most frequently used methodologies for the study of the processes of social and professional integration of people with disabilities in Spain. In order to accomplish that goal a revision of existing studies on disability in Spain was made. During this revision an analysis of the techniques used (quantitative and qualitative) and the different theoretical frameworks used (concepts of disability and social exclusion) was carried out. The main findings indicate a predominance of qualitative and mixed methods analysis, a multidimensional approach and a bio-psychosocial model of disability.

\section{Keywords}

Disability, social research

methodology, functional diversity, social inclusion, labour inclusion, data sources.

\author{
Antonia Sánchez Alcoba \\ <antonia.sanchez3@um.es> \\ Universidad de Murcia
}

\section{Manuel Hernández Pedreño} <manuel@um.es>

Universidad de Murcia

\section{Mariano Imbernón}

Fernández-Henarejos <mariano.imbernon@um.es>

Universidad de Murcia

\begin{abstract}
Para citar:
Sánchez, A. et al. (2016): "Estrategias metodológicas para el estudio de la inserción sociolaboral de las personas con discapacidad", Revista Española de Discapacidad, 4 (I): 97-II7.
\end{abstract}

Doi: <http://dx.doi.org/IO. 55 69/23405 I04.04.01.06>

Fecha de recepción: 22-02-20I6 Fecha de aceptación: $08-06-2016$ 


\section{Introducción}

El presente trabajo trata de analizar las estrategias metodológicas aplicadas en los estudios sobre los procesos sociolaborales de la población con discapacidad o diversidad funcional ${ }^{\mathrm{r}}$, identificando sus limitaciones $\mathrm{y}$ potencialidades, abarcando tanto el diseño de la propia investigación como la participación en ella de los distintos agentes que intervienen en la inserción sociolaboral (empresarios, formadores, familia, etc.), así como la propia persona con discapacidad.

La diversidad funcional es inherente a la propia existencia de la humanidad. Las capacidades funcionales de la persona han sido un criterio de medición y clasificación del individuo atribuyéndole una identidad individual y social. Esta construcción social de la discapacidad se ha definido en consonancia a los valores socioculturales de cada momento histórico. Sin embargo, en los albores del siglo XXI todavía es latente la discapacidad como causa de desigualdad social, a pesar del avance legislativo actual.

Esta desigualdad de la población con discapacidad conlleva una constante situación de inferioridad y es transversal a todas las áreas de la vida. El nivel económico, el nivel educativo, las tasas de empleo, aspectos sociosanitarios, el hábitat, las relaciones sociales y la ciudadanía de las personas con discapacidad siempre se muestran inferiores respecto a la población sin discapacidad. Todas las dimensiones influyen y determinan los contextos individuales y colectivos. No obstante, el ámbito laboral ejerce un gran peso en las sociedades actuales, influyendo directamente sobre la renta, el acceso a los recursos y una integración social plena.

I. En este artículo se emplean los términos de discapacidad y diversidad funcional de forma equivalente para referirse a la persona con discapacidad. En la actualidad, esta cuestión conceptual está en debate por el carácter peyorativo tanto del significado como del significante del prefijo "dis".
A este escenario ha de unirse el desfavorable contexto socioeconómico de estos últimos años, que ha aumentado las situaciones de vulnerabilidad y exclusión social en la población activa, siendo de mayor incidencia en la población con discapacidad. A pesar de los importantes logros conseguidos en el acceso al empleo de este colectivo, el camino para alcanzar la integración sociolaboral de las personas con discapacidad está inconcluso y, actualmente, estancado. En España, el interés empírico por la inserción sociolaboral de las personas con discapacidad se hace patente desde algunas operaciones estadísticas estatales, desde publicaciones e investigaciones al respecto de instituciones del tercer sector o investigadores vinculados al estudio de este colectivo, como el Comité Español de Representantes de Personas con Discapacidad (CERMI), desde la creación de centros específicos de análisis, como el Observatorio sobre Discapacidad y Mercado de Trabajo en España (ODISMET), o desde algunas áreas universitarias.

\section{Objetivos y metodología}

El fin de este trabajo es el análisis de las estrategias metodológicas más utilizadas para conocer los procesos de inserción sociolaboral de la población con discapacidad, revisando las debilidades y fortalezas. La multidimensionalidad y la complejidad del fenómeno de la discapacidad hace necesario valorar la adecuación de las distintas técnicas de investigación social empleadas, ya sean cuantitativas y cualitativas.

A nivel epistemológico, toda investigación social se enmarca en un paradigma determinado, en el que se configuran las teorías y metodologías empleadas. Esta conexión hace necesario realizar dos tipos de revisiones en los estudios sobre discapacidad, una teórica y otra empírica, siendo ambas la base metodológica de este trabajo. La revisión teórica comprende un análisis de los conceptos y tipologías, modelos sociales 
contemplados y las dimensiones consideradas. Respecto a la revisión empírica cuantitativa, el análisis se ha dirigido a la revisión y valoración de las diferentes estadísticas nacionales sobre discapacidad, que aportan información laboral y operaciones estadísticas del mercado laboral con información sobre discapacidad. Principalmente, se ha centrado en la operación estadística 'El Empleo de las Personas con Discapacidad' (EPD), del Instituto Nacional de Estadísticas (INE), junto a diversas investigaciones al respecto de otras entidades o profesionales.

Por su parte, la revisión cualitativa ha consistido en el análisis de estudios que ofrecen información relacionada con la inserción laboral de este colectivo. En particular, se ha valorado el rol desempeñado por los sujetos que son objeto de la investigación; el tipo de diseño de investigación; las dimensiones consideradas en la investigación; la entidad o entidades financiadoras; los agentes implicados en el proceso de investigación (familia, profesionales, etc.); las instituciones que inciden en la inserción laboral (administración, empresas, sindicatos, tercer sector, etc.); entre otros.

No obstante, el análisis no se reduce a la dimensión laboral, sino también a otras dimensiones directamente vinculadas con el mercado de trabajo, como pueden ser el nivel formativo o la situación económica, en consonancia con la multidimensionalidad de la inserción sociolaboral de las personas con discapacidad.

La revisión se ha estructurado siguiendo la tipología de los cuatro grandes grupos de discapacidad: física, sensorial, intelectual y mental, establecida por la Convención Internacional sobre los Derechos de las Personas con Discapacidad (ONU, 2006) y en relación con la Clasificación Internacional del Funcionamiento, CIF (OMS, 200I)2.

2. Se entiende por discapacidad física toda aquella deficiencia del sistema osteoarticular, neuromuscular, cardiovascular, inmunológico, respiratorio, digestivo, metabólico, endocrino entre otros; discapacidad sensorial toda deficiencia visual, auditiva y dificultades en la comunicación y el lenguaje; discapacidad intelectual toda disminución de las funciones mentales

\section{El marco teórico de la investigación social en discapacidad}

Los referentes conceptuales y los diferentes posicionamientos teóricos de partida en una investigación pueden condicionar la elección de la estrategia metodológica, pues pueden influir en la búsqueda y selección de datos primarios y secundarios, así como en la consideración de los agentes implicados. En materia de discapacidad, la propia definición y los modelos de inserción social han evolucionado en estas últimas décadas con importantes avances, en aras de un mejor conocimiento del fenómeno de la discapacidad y hacia una integración social más real de este colectivo. Dada la importancia del marco teórico en las metodologías, este apartado tiene el objetivo de ofrecer una revisión conceptual y teórica sobre la discapacidad y la exclusión social, dado el riesgo de exclusión que presenta este colectivo.

La discapacidad viene siendo objeto de estudio de la sociología desde finales del pasado siglo aunque, en nuestro país, más recientemente (Ferreira, 20Io). Inicialmente, la discapacidad era entendida como una cuestión de beneficencia y fundamentalmente sanitaria, obviando los factores sociales generadores de discapacidad. La consideración de la dimensión social de la discapacidad ha favorecido la apertura de líneas de investigación social, contribuyendo a un mayor entendimiento de la construcción social de este fenómeno y asumiendo las responsabilidades colectivas al respecto, por parte de las instituciones y de la sociedad en general.

Los primeros debates y estudios han sido impulsados por las entidades de la discapacidad, por algunos investigadores o profesionales próximos a la temática y por el propio colectivo; así como por el CERMI, el Real Patronato sobre Discapacidad o el Movimiento de Vida Independiente. Estas iniciativas y

superiores como inteligencia, lenguaje, aprendizaje y, una disminución de las funciones motoras; y discapacidad mental las alteraciones neurológicas y psicóticas. 
reivindicaciones han despertado el interés en la comunidad universitaria y en entidades tanto públicas como privadas, favoreciendo la fusión de diversas fuerzas y un progresivo avance en materia de discapacidad, algunos ejemplos de ello serían ODISMET, el Observatorio de Inserción Profesional y Asesoramiento Laboral (OPAL) de la Universidad de Valencia, el Centro de Estudios sobre Discapacidad y Promoción de la Autonomía Personal (DIPAD) y el Observatorio de la Exclusión Social (OES), ambos pertenecientes a la Universidad de Murcia, y diversos docentes y profesionales que promueven investigaciones en este campo.

\subsection{Concepto de discapacidad}

La discapacidad es un fenómeno diverso, multidimensional y complejo que presenta dificultades conceptuales, determinando problemas de medición, pues no todos los estudios, registros y estadísticas denominan a la discapacidad bajo los mismos términos, midiendo en muchos casos realidades distintas, como señala Huete (20I3). Estas deficiencias conceptuales han sido ampliamente analizadas por diversos autores (Jiménez Lara, 2007; Huete, 20I2; Colectivo IOE, 20I2).

En este sentido es fundamental destacar el papel de la Organización Mundial de la Salud (OMS) en la evolución de la clasificación de las discapacidades. Es destacable su segunda Clasificación Internacional del Funcionamiento (CIF) en $200 \mathrm{I}$, ya que aportó una sistematización de las discapacidades desde una perspectiva biológica, individual y social, fundamentada en diversos componentes sociosanitarios y no solo en las consecuencias de las deficiencias. De igual modo, propuso un enfoque bio-psico-social donde estos tres elementos jugarían un papel codeterminante, sin priorizar ninguno de ellos sobre los demás (Ferreira, 2008). Este planteamiento permite analizar la discapacidad como una construcción social, pues son las estructuras y los procesos sociales los que finalmente determinarán la concepción, valoración y posición social de las personas con diversidad funcional.
En la actualidad se define a las personas con discapacidad como "aquellas que tengan deficiencias físicas, mentales, intelectuales o sensoriales a largo plazo que, al interactuar con diversas barreras, puedan impedir su participación plena y efectiva en la sociedad, en igualdad de condiciones con las demás” (ONU, 2006: 4). En relación a este actual planteamiento conceptual, cabe mencionar el debate establecido por el propio colectivo hacia el término de diversidad funcional en sustitución por el de discapacidad, cuestión referida anteriormente en la introducción de este trabajo ${ }^{3}$.

Otro elemento de interés en el entendimiento de la discapacidad es el grado de severidad. El marco legislativo español considera "personas con discapacidad aquellas a quienes se les haya reconocido un grado de discapacidad igual o superior al 33 por ciento", recogido en el Real Decreto Legislativo I/2013, de 29 de noviembre, por el que se aprueba el Texto Refundido de la Ley General de derechos de las personas con discapacidad y de su inclusión social. De igual modo, este aspecto legislativo constituye un criterio básico en la contratación de personas con discapacidad para esta tipología de contrato.

\subsection{Concepto de exclusión social}

Las personas con discapacidad experimentan situaciones de desigualdad y en detrimento de la dignidad, teniendo serias dificultades para lograr su autonomía personal (Anaut et al., 20I4: 2). En esta línea, Díaz (20I I) plantea que la discapacidad implica más una situación de desigualdad que de exclusión social. Ciertamente, tener una deficiencia no implica una situación de exclusión. Sin embargo, la discapacidad sí está vinculada a la exclusión social debido a su vulnerabilidad constante pues los hogares con presencia de discapacidad

\footnotetext{
3. Si se desea profundizar en el enfoque de la diversidad funcional se puede consultar a Palacios y Romañach (2008) o a Almeida et al. (2010).
} 
presentan menores ingresos y la persona con discapacidad asume el coste adicional de la misma (Anaut y Arza, 2015). Además, un segmento significativo de este colectivo vive en situación de exclusión.

En 2008 , un $14,8 \%$ de personas con discapacidad vivían en la pobreza extrema y un $36,8 \%$ en la pobreza relativa (Huete, 2013 ). Aunque el impacto de la crisis ha sido mayor en la población sin discapacidad, los niveles de pobreza y exclusión para las personas y hogares con discapacidad se han mantenido por encima del conjunto de la población. Esta situación, unida al incremento de la pobreza moderada de los hogares con discapacidad, de un $9 \%$ en 2009 a un 20,6\% en 20I3 (Anaut y Arza, 20I5: I4), constata el estado de vulnerabilidad y desigualdad para este colectivo. No obstante, el trabajo de Anaut y Arza no considera el impacto de la crisis en función de la edad, ya que la población con discapacidad aumenta a partir de los 65 años; disponiendo más este segmento de edad de la protección de las pensiones de jubilación y siendo, por tanto, las repercusiones de la crisis muy diferentes por grupos etarios.

Desde un análisis comparativo de las políticas públicas nacionales sobre discapacidad (I982-20I4), se puede establecer que a pesar del avance en materia legislativa la realidad de este colectivo refleja especiales dificultades de acceso a la plena participación e igualdad social (Cano, Díaz, Ferreira, Rodríguez y Toboso, 20I5).

El concepto de exclusión social, entendido como un proceso multidimensional y como el resultado de la acumulación de desventajas sociales en siete áreas vitales (ingresos, educación, trabajo, salud, vivienda, relaciones sociofamiliares y participación) (Hernández, 20IO), puede contribuir al análisis de los procesos de inserción sociolaboral de la persona con discapacidad, pues la trayectoria sociolaboral del individuo está relacionada con todas las dimensiones.
La situación de desigualdad de la población con discapacidad obliga a considerar el Enfoque de los Derechos Humanos (EDH) en el entendimiento de la discapacidad, establecido sobre el principio universal de que la persona es perceptora de derechos y obligaciones y no como objeto de caridad (Raya, Caparrós y Peña, 20I 2; Anaut y Arza, 20I 5). Esta línea argumental está vinculada a la Convención Internacional de los derechos de las personas con discapacidad (ONU, 2006), con el objetivo de garantizar la ciudadanía de pleno derecho para este colectivo.

\subsection{Modelos de inserción de las personas con discapacidad}

Históricamente, la consideración social de la discapacidad se ha construido a partir de las connotaciones peyorativas asociadas al fenómeno. En la Antigüedad, la persona portadora de alguna deficiencia física o psíquica no era considerada de la especie humana (Sanmartín, I998), cuestión que evolucionó en la Edad Media hacia una situación de exclusión y marginalidad de la persona (Garvía, I997); esta coyuntura de aislamiento social comenzó a dar síntomas de cambio a finales del siglo XX, cuando se adquirió la concepción de personas con discapacidad, aunque sin plenitud de derechos y en una posición de desventaja social. A partir de estas valoraciones, y una vez expuesto este breve recorrido histórico, se definen los modelos de inserción de las personas con discapacidad, como muestra el cuadro I.

El modelo predominante en España hasta finales del siglo pasado ha sido el modelo médico-rehabilitador, centrado en el tratamiento y rehabilitación hacia la "normalidad" como elemento de inclusión, que entendía la discapacidad como la consecuencia de una enfermedad donde el individuo es el único responsable (Ferreira, 2008). En oposición a este modelo, surge en los años ochenta el modelo social, de la mano de la sociología, que se hizo eco de las reivindicaciones del propio colectivo para ser considerados personas de pleno derecho 


\begin{tabular}{|l|l|l|}
\hline \multicolumn{1}{|l}{ Cuadro 1. Modelos de inserción de la discapacidad y postulados } \\
\hline Contexto & Modelos/Paradigmas & Características \\
\hline \multirow{5}{*}{ S. XIX y S. XX } & Médico-Rehabilitador & $\begin{array}{l}\text { Mundo sanitario } \\
\text { Únicamente factores biológicos }\end{array}$ \\
\cline { 2 - 3 } & Social & $\begin{array}{l}\text { Len Barton } \\
\text { Sólo factores sociales }\end{array}$ \\
\hline \multirow{5}{*}{ S. XXI } & BIO-PSICO-SOCIAL & $\begin{array}{l}\text { OMS, 2001 } \\
\text { Factores biológicos y psicosociales } \\
\text { Referente actual }\end{array}$ \\
\cline { 2 - 3 } & Diversidad Funcional & $\begin{array}{l}\text { Modelo de Diversidad: sociedad diversa } \\
\text { Movimiento de Vida Independiente }\end{array}$ \\
\cline { 2 - 3 } & Paradigma de Autonomía Personal & $\begin{array}{l}\text { A. Jiménez Lara } \\
\text { Centrado en el entorno }\end{array}$ \\
\cline { 2 - 3 } & Político-Activista & Colectivo discriminado por la sociedad \\
\cline { 2 - 3 } & Universal & Concepción universal de la discapacidad \\
\hline
\end{tabular}

Fuente: elaboración propia

(Barton, 2008). Este modelo se centra exclusivamente en los factores sociales de la discapacidad y considera que las causas que originan la discapacidad son las mismas que dan lugar a procesos de exclusión social en otros ámbitos de la vida (Colectivo IOE, 20I2).

De la segunda clasificación de la OMS en $200 \mathrm{I}$ sobre las discapacidades (CIF), mencionada anteriormente, emana el modelo bio-psicosocial, que engloba tres niveles de valoración: un nivel biológico, en cuanto a la existencia de una deficiencia; un nivel individual, referido a las actividades y limitaciones de la persona (funcionamiento); y un nivel social, donde el contexto social cobra gran relevancia (Ferrante y Ferreira, 2010). En este sentido, cabe mencionar la aportación de Díaz Velázquez (2009) que, no coincidiendo con la perspectiva de Jiménez Lara (2007) y otros autores que señalan que este nuevo modelo biopsicosocial es una síntesis de los modelos médico y social, plantea que este nuevo paradigma responde a una concepción aún médica o rehabilitadora de la discapacidad pues, a pesar de asumir la incidencia de los factores contextuales en la emergencia y el desarrollo de la discapacidad, sigue centrando el núcleo de análisis y de intervención exclusivamente en el individuo (Díaz Velázquez, 2009).
Paralelamente se desarrolla el modelo de diversidad funcional, vinculado a los movimientos de vida independiente, que insiste en suprimir la discriminación hacia este colectivo (Ferreira, 2010). Promueve la incorporación de los derechos humanos y el fomento de la autonomía (Palacios y Romañach, 2006: I I I). Desde otra perspectiva, Jiménez Lara (2007) plantea el Paradigma de Autonomía Personal, que centra el problema en las circunstancias y el entorno. Esta tesis establece que el contexto es quien discapacita a la persona y no su deficiencia individual pues, frecuentemente, ésta es causada o agravada por el propio entorno (Díaz Velázquez, 20I0).

Se pueden destacar otros dos modelos que coexisten con los modelos anteriores y comparten los aspectos sociales de la discapacidad. Por un lado, el modelo politico-activista, basado en la acción política y social, considerando que la persona con discapacidad forma parte de un microgrupo minoritario discriminado por la propia sociedad (Minguijón et al., 2005). Por otro lado, el modelo universal basado en la idea de la discapacidad como un hecho universal y que plantea que las políticas deben ser universales, dirigidas al conjunto de la población, pues la discapacidad es un elemento intrínseco de la humanidad y no un rasgo diferenciador entre la población (Minguijón et al., 2005). 


\section{Análisis de fuentes secundarias para el estudio de la inserción sociolaboral de las personas con discapacidad}

Las fuentes oficiales disponibles en España que permiten estudiar la relación entre discapacidad y empleo se pueden dividir en dos grandes grupos. Por un lado, las fuentes exclusivas sobre discapacidad y, por otro lado, las fuentes propias de empleo que incluyen la discapacidad como una variable transversal.

\subsection{Fuentes estadísticas sobre discapacidad con información laboral}

El INE ha realizado en su historia reciente diversas encuestas sobre la población con discapacidad. Estas operaciones estadísticas cuentan con una gran amplitud muestral en nuestro país, pues así garantizan la representatividad de la submuestra del colectivo de personas con discapacidad. El cuadro 2 resume las características de estas encuestas.

La Encuesta sobre Deficiencias, Discapacidad y Minusvalías (EDDM, I986) significó la disposición de los primeros datos cuantitativos a nivel nacional, las características sociodemográficas y las tendencias generales de este colectivo. Posteriormente, con la realización de la Encuesta de Discapacidad, Deficiencias y Estado de Salud (EDDS, I999), se incorporaban elementos relativos al estado de salud y un acercamiento a las circunstancias que conlleva la situación de tener una discapacidad. En ambas operaciones estadísticas ha existido el problema de la definición del concepto de discapacidad y la necesidad de homogeneizar las distintas tipologías. La EDDM-86 y la EDDS99 se elaboraron siguiendo la metodología de la Clasificación Internacional de Deficiencias, Discapacidad y Minusvalía (CIDDM), propuesta por la OMS en I980. Tras las críticas vertidas al enfoque excesivamente médico de la CIDDM, que obviaba el contexto social en el que se desenvuelve el individuo, se recomendó la adopción de la CIF con una perspectiva mucho más holística y multidimensional.

El siguiente serial es la Encuesta sobre Discapacidad, Autonomía personal y situaciones de Dependencia (EDAD-2008), proporcionando una base estadística para el seguimiento de la autonomía personal y la prevención de las situaciones de dependencia en el Sistema Nacional de Dependencia y la Ley de la Dependencia (2006). Esta macroencuesta fue la primera en utilizar la CIF, aunque tan sólo parcialmente, y con ello permite la comparabilidad con las encuestas anteriores. La importancia de esta labor estadística reside en la valoración del propio colectivo sobre los ítems planteados, que incluyen entre otras, cuestiones

\begin{tabular}{|c|c|c|c|}
\hline Denominación & Año & $\begin{array}{l}\text { Dimensiones de } \\
\text { análisis }\end{array}$ & Marco conceptual \\
\hline $\begin{array}{l}\text { Encuesta sobre Deficiencias, } \\
\text { Discapacidad y Minusvalía (EDDM) }\end{array}$ & 1986 & Salud & \multirow{2}{*}{$\begin{array}{l}\text { Clasificación Internacional de } \\
\text { Deficiencias, Discapacidad y } \\
\text { Minusvalía (CIDDM) (OMS, 1980) } \\
\text { Modelo Médico-Rehabilitador }\end{array}$} \\
\hline $\begin{array}{l}\text { Encuesta de Discapacidad, } \\
\text { Deficiencias y Estado de Salud } \\
\text { (EDDS) }\end{array}$ & 1999 & Salud, Relacional & \\
\hline $\begin{array}{l}\text { Encuesta sobre Discapacidades, } \\
\text { Autonomía personal y situaciones } \\
\text { de Dependencia (EDAD) }\end{array}$ & 2008 & \multirow{2}{*}{$\begin{array}{l}\text { Salud, Laboral, } \\
\text { Economía, Formativa, } \\
\text { Relacional, } \\
\text { Participación }\end{array}$} & \multirow{2}{*}{$\begin{array}{l}\text { Clasificación Internacional } \\
\text { del Funcionamiento, de la } \\
\text { Discapacidad y la Salud (CIF) } \\
\text { (OMS, 2001) } \\
\text { Modelo Bio-psico-social }\end{array}$} \\
\hline $\begin{array}{l}\text { Encuesta sobre Integración Social y } \\
\text { Salud (EISS) }\end{array}$ & 2012 & & \\
\hline
\end{tabular}

Fuente: elaboración propia 
sobre las barreras en su participación social y también en el mercado laboral.

Por otro lado, está la Encuesta sobre Integración Social y Salud (EISS, 201 2$)^{4}$, proporcionando una explotación cuantitativa sobre la interacción entre la condición de salud y la integración social de la población española, siendo comparable a nivel europeo, pues incorpora plenamente la CIF. En este sentido, permite estudiar los distintos ámbitos de la vida social de la población con discapacidad y, al mismo tiempo, se nutre del enfoque biopsicosocial con la valoración de los apoyos técnicos y asistenciales.

En la producción científica, son varios los autores que reflejan un análisis de la realidad laboral de la población con discapacidad en base a la idoneidad de las fuentes oficiales cuantitativas secundarias, en especial la EDAD2008. El estudio del Colectivo IOE (20 I 2) sobre la inclusión de la población española con discapacidad centra su análisis en la EDAD que, entre otras dimensiones, aborda los aspectos laborales de este colectivo; a su vez, esta fuente se compara con sus dos antecesoras, la EDDM86 y EDDES-99, evidenciando las limitaciones de medición. Las encuestas no mantienen los mismos parámetros, impidiendo con ello la comparación entre ambas. La primera encuesta de 1986 reflejaba algunas limitaciones muy frecuentes en las personas mayores, mientras que en la segunda encuesta de I 999 no se consideraban estos aspectos. Muestra de ello, y valga como ejemplo, en la segunda encuesta no se incluyó el ítem de "no poder correr a paso gimnástico $50 \mathrm{~m}$ ”, cuando eran cuatro millones de personas las que se veían afectadas por esta limitación. La consecuencia más inmediata fue no reflejar el aumento real de la prevalencia sobre discapacidad pues, coyunturalmente,

4. No obstante, por sus características, la Encuesta sobre Integración Social y Salud (EISS, 20I2) no se puede considerar como la última de una serie de macroencuestas sobre discapacidad realizadas por el INE. Así, el propio INE no la incorpora como tal. De hecho, el Programa anual 2016 del Plan Estadístico Nacional 2013-20I6 contempla entre sus acciones los preparativos para la nueva macroencuesta "de Discapacidad, Autonomía Personal y Situaciones de Dependencia”: http:// www.boe.es/boe/dias/2015/12/28/pdfs/BOE-A-2015-14209.pdf la tendencia general del envejecimiento de la población debía producir un incremento mayor de la tasa de discapacidad.

Por otro lado, Huete (20I3) en su trabajo sobre la exclusión de la población con discapacidad en España utiliza en su análisis la Encuesta Social Europea de 2010 (ESE), desde un enfoque multidimensional de la exclusión social, incluyendo entre otras dimensiones el empleo. Esta encuesta bianual es un estudio comparativo y longitudinal en la que participan una treintena de países europeos, entre los que figura España, y se le atribuye el valor de estudiar la evolución de las actitudes, atributos y comportamientos sociales y políticos de los ciudadanos europeos; sin embargo, presenta la limitación en la fiabilidad muestral para estudios complejos, pues la muestra fue de 286 casos y la media por país no llegó a diez encuestas.

\subsection{Fuentes estadísticas sobre el mercado laboral con información sobre discapacidad}

Este tipo de fuentes comprende los dos módulos ad hoc de la EPA relativos a la discapacidad, junto con las dos operaciones estadísticas sobre discapacidad en materia de empleo, el Empleo de las Personas con Discapacidad (EPD) y el Salario de las Personas con Discapacidad (SPD), cuyas características se muestran en el cuadro 3 .

La incorporación del módulo ad hoc de la EPA del 2002 fue en base a la normativa comunitaria del Sistema Estadístico Europeos. En la EPA de 20I I se cambió la denominación de "Empleo de las personas con discapacidad", según el Reglamento 6 , por un módulo titulado "Problemas de salud y su relación con el empleo", y con ello evitar las posibles confusiones con el EPD. A diferencia del módulo de 2002, el de 20 I I pretende ajustarse a la concepción de la CIF al preguntar por los problemas de salud y por las actividades básicas de manera que todos los encuestados

\footnotetext{
5. Reglamento de la Comisión I 566/200 I de I 2 de julio. 6. Reglamento de la Comisión 3 I 7/2010 de I 6 de abril de 2010.
} 


\begin{tabular}{|c|c|c|c|c|}
\hline Tipo & Denominación & Periodo & Identificación del colectivo & Población muestral \\
\hline $\begin{array}{l}\text { Módulo ad } \\
\text { hoc EPA, } \\
2003\end{array}$ & $\begin{array}{l}\text { Personas con } \\
\text { discapacidad y } \\
\text { su situación en el } \\
\text { empleo }\end{array}$ & $\begin{array}{l}2002,2^{\circ} \\
\text { trimestre }\end{array}$ & No CIF. & Población 15-64 años \\
\hline $\begin{array}{l}\text { Módulo ad } \\
\text { hoc EPA, } \\
2012\end{array}$ & $\begin{array}{l}\text { Problemas de salud } \\
\text { y su relación con el } \\
\text { empleo }\end{array}$ & $\begin{array}{l}\text { 2011, cuatro } \\
\text { trimestres }\end{array}$ & $\begin{array}{l}\text { CIF, pregunta por los } \\
\text { problemas de salud y por las } \\
\text { actividades básicas. }\end{array}$ & $1 / 6$ de la muestra EPA \\
\hline Tipo & Denominación & Periodo & Identificación del colectivo & Fuentes combinadas \\
\hline $\begin{array}{l}\text { Operación } \\
\text { estadística. } \\
\text { EPA, } 2014\end{array}$ & $\begin{array}{l}\text { El Empleo de las } \\
\text { Personas con } \\
\text { Discapacidad (EPD) }\end{array}$ & $\begin{array}{l}\text { Anual, } \\
\text { disponible } \\
2009-2013\end{array}$ & $\begin{array}{l}\text { - } \text { Concepto legal de } \\
\text { discapacidad (igual o } \\
\text { superior al } 33 \% \text { ). } \\
\text { - } \\
\text { No permite la comparación } \\
\text { con las mediciones } \\
\text { basadas en la CIF. }\end{array}$ & $\begin{array}{l}\text { Base de Datos Estatal } \\
\text { de Personas con } \\
\text { Discapacidad (BEPD), } \\
\text { Tesorería General de } \\
\text { la Seguridad Social } \\
\text { (TGSS), Instituto } \\
\text { Nacional de Seguridad } \\
\text { Social (INSS), Sistema } \\
\text { para la Autonomía } \\
\text { y Atención a la } \\
\text { Dependencia (SAAD) }\end{array}$ \\
\hline $\begin{array}{l}\text { Operación } \\
\text { estadística. } \\
\text { SPD, } 2013\end{array}$ & $\begin{array}{l}\text { El Salario de las } \\
\text { Personas con } \\
\text { Discapacidad (SPD) }\end{array}$ & $\begin{array}{l}\text { Anual, } \\
\text { disponible } \\
\text { 2010-2011 }\end{array}$ & 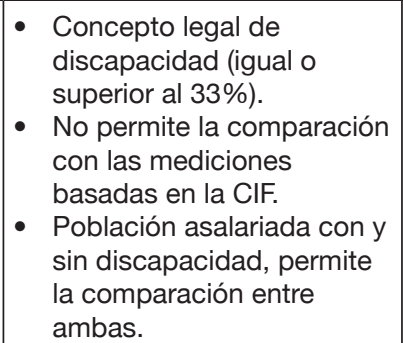 & $\begin{array}{l}\text { Encuesta de Estructura } \\
\text { Salarial, Base de Datos } \\
\text { Estatal de Personas } \\
\text { con Discapacidad } \\
\text { (BEPD) y Tesorería } \\
\text { General de la Seguridad } \\
\text { Social (TGSS) }\end{array}$ \\
\hline
\end{tabular}

Fuente: elaboración propia.

respondieran separadamente a ambos tipos de cuestiones.

El carácter puntual y espaciado en el tiempo de las principales operaciones estadísticas que aportan datos de discapacidad y mercado laboral hacía necesario algún tipo de operación estadística que con carácter periódico aportara información actualizada sobre la evolución del empleo entre las personas con discapacidad. Fruto de la colaboración de distintas organizaciones sociales e institucionales ${ }^{7}$, el INE estableció dos operaciones estadísticas con periodicidad anual el EPD y el SPD, siendo el cruce de los ficheros estadísticos extraídos de

7. En el grupo técnico de trabajo participaron el CERMI, la Fundación Once, el IMSERSO y la entonces Dirección General de Coordinación de Políticas Sectoriales sobre Discapacidad. encuestas con distintos registros de carácter administrativo la novedad respecto a otras encuestas sobre el empleo.

La integración de datos entre distintas fuentes tiene la ventaja de reducir la sobrecarga estadística y reducir costes. Por el contrario, tiene la limitación de que al cruzar los datos con fuentes administrativas el enfoque no puede ser sino legalista, por lo que el fenómeno multidimensional de la discapacidad no puede ser abordado homogéneamente con respecto a las otras macroencuestas del INE.

El EPD proporciona información laboral de individuos y de hogares cruzada por distintas variables sociodemográficas: grado y tipo de discapacidad, fomento del empleo, percepción 
de prestaciones y situación de dependencia. El periodo de referencia es el año natural y los datos vienen expresados en medias anuales. La población a la que va referida la operación son las personas en edad laboral, con certificado de discapacidad ${ }^{8}$ y que residen en viviendas familiares principales, por lo que los datos registrados de la BEPD, base de datos estadística con la que se cruza, residentes en establecimientos colectivos no se han considerado.

Respecto al SPD, señalar que el periodo de referencia es con carácter general el año natural excepto para las ganancias mensuales donde la referencia es el mes de octubre. Se proporcionan datos de ganancias, distribución y diferencias salariales, tanto para la población con o sin discapacidad, que facilita la comparación cruzada por distintas variables sociolaborales. Los datos están referidos a trabajadores asalariados por cuenta ajena. Sin embargo, y a diferencia de lo que sucede con el EPD, la muestra no cuenta con un número suficiente de individuos con discapacidad para mostrar datos territorialmente desagregados.

Una limitación común en el EPD y el SPD es que parten de un concepto de discapacidad legal y en función del reconocimiento de grado, igual o superior al $33 \%$, que no se adapta a la CIF, por lo que no se recoge el fenómeno en toda su amplitud como sería deseable, y como se puede observar en el cuadro 3. Por otro lado, tienen la ventaja de ofrecer la posibilidad de realizar la evaluación de las distintas medidas para la inserción laboral y el fomento del empleo, al tomar como referencia el grado de discapacidad.

Otro aspecto a destacar es la información proporcionada por los organismos responsables de las políticas de empleo. El Servicio Público de Empleo Estatal (SEPE) publica periódicamente con carácter anual las cifras de contratación de personas con certificado de discapacidad, tanto a nivel nacional como provincial, aunque el registro presenta la limitación de no mostrar

8. Grado de discapacidad superior o igual al $33 \%$, según el procedimiento establecido por RD I97I/I 999 modificado por RD I364/20I2. el total de personas con discapacidad, pues su indicación no es de obligada cumplimentación; este aspecto dificulta la segregación de los datos (SEPE, 20I 5 ). De igual modo, tampoco es obligatoria la indicación de la tipología en los registros de demandantes de empleo. Frente a ello, los registros administrativos presentan la ventaja de ofrecer los datos de contratación y demandantes desglosados territorialmente inferiores a nivel provincial.

Las operaciones estadísticas analizadas pueden constituir una primera fase de análisis de las fuentes cuantitativas. De modo complementario a la revisión aplicada, se pueden tener en cuenta otras encuestas que contemplan a las personas con discapacidad, como la Encuesta de Condiciones de Vida, la Muestra Continua de Vidas Laborales, entre otras. Del mismo modo, también cabe mencionar el primer informe de ODISMET sobre empleo y discapacidad (ODISMET, 20I4).

\section{Análisis de la idoneidad de las diversas técnicas de investigación social para el estudio de la inserción sociolaboral de las personas con discapacidad}

En este apartado se realiza una revisión de la aplicación de las diversas técnicas de investigación social, como fuente primaria de investigación, aplicadas al estudio de la inserción sociolaboral de las personas con discapacidad. En líneas generales, la producción es mayoritariamente cualitativa, que podría ser aludido a los diseños muestrales y/o criterios económicos.

Toda técnica de investigación social posee sus ventajas y limitaciones, su valor reside principalmente en la idoneidad para obtener una determinada información, y no en la superioridad de una sobre otra. Las técnicas cuantitativas permiten medir datos numéricos sobre la realidad social de la población con discapacidad, aportan las características y 
tendencias sociodemográficas y permiten el contraste con la población sin discapacidad. Por su parte, las técnicas cualitativas son idóneas para conocer las trayectorias, las construcciones ideológicas, los procesos de inserción sociolaboral, además de aportar luz al desconocimiento subjetivo de la realidad social de las personas con discapacidad.

Resulta importante señalar, que gran parte de los estudios sobre discapacidad son promovidos principalmente por entidades privadas y vinculadas con la discapacidad, aunque en su mayoría a través de fondos públicos. Desde las ciencias sociales los estudios de corte psicológico son los de mayor producción, seguidos del ámbito de la educación, del trabajo social y del área jurídica; mientras los estudios sociológicos sobre discapacidad son relativamente recientes en su producción literaria y empírica; finalmente existen algunas aproximaciones antropológicas a la discapacidad, aunque no desde el análisis laboral.

En cuanto a la tipología de la discapacidad analizada, predominan los estudios de perspectiva general, que engloban a los cuatros grandes grupos de la discapacidad (física, sensorial, intelectual y mental). Además, en todos los estudios examinados se considera el grado de multidimensionalidad contemplado; ya sea de forma explícita o implícita, es decir, contempladas directa o indirectamente, así por ejemplo, la dimensión trabajo es explicita, mientras los ingresos y la formación se desarrollan indirectamente y en menor profundidad.

Desde una mirada específica en relación al empleo, la mayoría de los trabajos versan sobre discapacidad física, intelectual y visual, seguidos de la discapacidad mental y auditiva. Por último, en la totalidad de los estudios está presente y de manera indispensable, la colaboración y apoyo tanto de las entidades como de las propias personas con discapacidad. En este sentido, cabe mencionar la metodología Investigación Acción y Participación (IAP) que promueven la participación de la población objeto de estudio en el propio proceso de investigación como sujetos activos de la misma (Colectivo IOÉ, I993).

\subsection{Encuesta}

España dispone de una importante información procedente de fuentes oficiales sobre discapacidad a través de las macroencuestas. Sin embargo, el interés y la demanda de estudios proceden, principalmente, de las entidades relacionadas con la discapacidad y del propio colectivo más que de las administraciones públicas y comunidad científica.

La encuesta presenta claras desventajas respecto a otras técnicas en su aplicación a la población con discapacidad, destacando los siguientes puntos: limitaciones en la delimitación de la muestra significativa (Toboso y Rogero, 20I2), dificultad de cumplimentar el cuestionario según el tipo de discapacidad, precisando la ayuda de terceros, y las barreras de accesibilidad (comunicativas, movilidad, desplazamiento, entre otras).

Algunas limitaciones de la encuesta personal cara a cara se ven reducidas al aplicar un cuestionario virtual, como se hizo en el estudio exploratorio "Aspectos psicosociales de la integración de personas con discapacidad en el mercado ordinario de trabajo" (Alcover y Pérez, 20Io), en el que se realizó una encuesta a I 35 trabajadores con discapacidad, incluyendo el cuestionario aspectos vinculados con los distintos apoyos que reciben estas personas.

\subsection{Entrevista en profundidad}

La técnica cualitativa de la entrevista es la más empleada en los estudios sobre discapacidad y mercado laboral, esta herramienta presenta la gran ventaja de una muestra de casos o perfiles reducida y, en diversos cosas, un menor coste. La entrevista permite captar vivencias y discursos que posibilitan una mayor comprensión de los procesos sociales. En el cuadro 4 , se ofrecen algunos ejemplos del uso de la entrevista en el estudio de este binomio de discapacidad e inserción laboral y sus principales características. 


\begin{tabular}{|c|c|c|c|}
\hline Título & $\begin{array}{l}\text { Autoría y } \\
\text { contexto }\end{array}$ & Muestra seleccionada & Aportaciones / Valoraciones \\
\hline $\begin{array}{l}\text { La mejora de las } \\
\text { condiciones de } \\
\text { trabajo y de vida de } \\
\text { las personas con } \\
\text { discapacidad }\end{array}$ & $\begin{array}{l}\text { Colecitvo IOÉ } \\
\text { (1997), España }\end{array}$ & $\begin{array}{l}11 \text { entrevistas: personas } \\
\text { con discapacidad, gerentes, } \\
\text { empresarios y profesionales. }\end{array}$ & $\begin{array}{l}\text { Dificultad de entrevistar al } \\
\text { perfil auditivo; se suplió con las } \\
\text { entrevistas al jefe de sección y el } \\
\text { técnico encargado del seguimiento } \\
\text { del caso. } \\
\text { Dimensiones de análisis: Laboral, } \\
\text { ingresos, formación, salud y } \\
\text { relacional. }\end{array}$ \\
\hline $\begin{array}{l}\text { Discapacidad y } \\
\text { Trabajo en España }\end{array}$ & $\begin{array}{l}\text { Colectivo IOÉ } \\
\text { (1998), España }\end{array}$ & $\begin{array}{l}44 \text { entrevistas agentes } \\
\text { próximos: } 15 \text { familia y } \\
\text { vecinos, } 9 \text { profesionales, } \\
12 \text { agentes laborales, } \\
8 \text { representantes de } \\
\text { asociaciones. }\end{array}$ & $\begin{array}{l}\text { Se complementa con historias de } \\
\text { vida y grupos de discusión } \\
\text { Dimensiones de análisis: todas. }\end{array}$ \\
\hline $\begin{array}{l}\text { Discapacidad y } \\
\text { empleo }\end{array}$ & $\begin{array}{l}\text { Miguijón et al. } \\
\text { (2005), Aragón }\end{array}$ & $\begin{array}{l}36 \text { entrevistas: } 15 \text { a } \\
\text { personas con discapacidad, } \\
8 \text { a representantes } \\
\text { de asociaciones de } \\
\text { discapacidad, } 2 \text { a } \\
\text { sindicalistas y } 11 \text { a } \\
\text { responsables de empresas. }\end{array}$ & $\begin{array}{l}\text { Se complementa con fuentes } \\
\text { cuantitativas secundarias } \\
\text { Dimensiones de análisis: laboral, } \\
\text { ingresos formación, relacional y } \\
\text { participación. }\end{array}$ \\
\hline $\begin{array}{l}\text { El impacto de la } \\
\text { crisis económica en } \\
\text { las personas con } \\
\text { discapacidad y sus } \\
\text { familias }\end{array}$ & $\begin{array}{l}\text { Huete et al. } \\
\text { (2009), España }\end{array}$ & $\begin{array}{l}7 \text { entrevistas a expertos: } \\
\text { directivos, representantes } \\
\text { y profesinales de } \\
\text { asociaciones con atención a } \\
\text { la discapacidad. }\end{array}$ & $\begin{array}{l}\text { Se emplean otras técnicas } \\
\text { cualitativas donde sí participan } \\
\text { personas con discapacidad: grupo } \\
\text { de discusión e historias de vida. } \\
\text { Dimensiones de análisis: todas. }\end{array}$ \\
\hline
\end{tabular}

Fuente: elaboración propia.

El trabajo del Colectivo IOÉ (I997) analiza la mejora de la situación sociolaboral de la población española con discapacidad. La investigación fue participativa pues, el trabajo de campo estaba dirigido hacia la propia persona con discapacidad (a excepción de la discapacidad física, con edades no superiores a los 35 años), y a los agentes relacionados con un centro de trabajo de la persona con discapacidad (gerentes, empresarios y profesionales). La limitación del estudio estuvo en la dificultad de entrevistar personalmente al perfil auditivo pues, normalmente requiere de un intérprete de lenguaje de signos o algún otro sistema de comunicación específico, cuestión que se suplió con las entrevistas al jefe de sección y el técnico encargado del seguimiento del caso. Este trabajo refleja un enfoque multidimensional completo (de las siete dimensiones), abordadas de una forma explícita en el diseño metodológico exceptuando la dimensión relacional y la participación, que se analizan indirectamente en los procesos de inserción laboral.

El estudio de la población española y aragonesa con discapacidad en torno al mercado laboral Minguijón et al. (2005), es otro claro ejemplo del uso de las entrevistas con una perspectiva humanista-cualitativa. El estudio se centra en el análisis interpretativo del discurso de las propias personas con discapacidad y de los agentes sociales más determinantes, donde no solo se abordó lo que decían, sino también cómo lo decían con el fin de identificar las dificultades y las ventajas para el acceso al mercado laboral y, estudiar las trayectorias de inserción. El análisis ha complementado la explotación e interpretación de fuentes secundarias cuantitativas con la información obtenida de las técnicas cualitativas. En este 
estudio, las entidades de inserción laboral tenían un papel clave como mediadores entre la oferta y la demanda, en la idoneidad del puesto de trabajo a la discapacidad, conocer las estrategias y optimización de itinerarios de inserción laboral desarrollados por las entidades. Este trabajo, además de la dimensión laboral del objeto de estudio, también alude indirectamente a las dimensiones formación, participación y relaciones sociales.

Otro ejemplo de aplicación de la entrevista en profundidad se localiza en la investigación sobre el impacto de la crisis económica Huete et al. (2009), estudio para la población con discapacidad y con la participación del colectivo en algunas técnicas cualitativas de las múltiples empleadas (entrevistas, grupos de discusión e historias de vida), además de entrevistas realizadas a expertos. Este estudio presenta una multidimensionalidad centrada en las esferas laboral, sociosanitaria y residencial de forma explícita; siendo el resto de dimensiones son abordadas implícita y parcialmente.

\subsection{Historia de vida}

La historia de vida aplicada a la discapacidad permite profundizar en la trayectoria vital de la persona, ofrece una perspectiva longitudinal, posibilita la identificación de las limitaciones y los apoyos en la inclusión sociolaboral experimentados y vividos desde el propio protagonista, además de compensar el objetivismo sociológico (Valles, I997; Pujadas, I992). Es habitual combinar esta técnica con la entrevista u otras técnicas cualitativas, como se muestra en el cuadro 5 .

El estudio multicualitativo de Huete et al (2009), desde una perspectiva sociológica, es un ejemplo de la combinación de técnicas cualitativas. Desde una perspectiva de género se aborda la tipología, la situación laboral y la existencia de cargas familiares, ofreciendo una visión multidimensional de la realidad sociolaboral de las personas con discapacidad.

La producción de estudios del Colectivo IOÉ también incluye una investigación multicualitativa, sobre los procesos de inclusión y exclusión sociolaborales de las personas española con discapacidad (Colectivo IOE, I998). En este trabajo, la información obtenida de otras técnicas cualitativas (entrevistas y grupos de discusión) permite profundizar y ampliar a las historias de vida, técnica central en este diseño metodológico. Por otro lado, la participación es inclusiva y centrada en el discurso del propio colectivo, junto a la participación de agentes sociales relacionados que complementan a los propios itinerarios de vida. Igual que en el estudio anterior revisado, éste emplea un enfoque multidimensional para el análisis de los procesos sociolaborales

Cuadro 5. Aplicación de la historia de vida al estudio de inserción sociolaboral de personas con
discapacidad
\begin{tabular}{|l|l|l|l|}
\hline Título & $\begin{array}{l}\text { Autoria/ } \\
\text { contexto }\end{array}$ & Muestras seleccionadas & Aportaciones / Valoraciones \\
\hline $\begin{array}{l}\text { Discapacidad y } \\
\text { Trabajo en España }\end{array}$ & $\begin{array}{l}\text { Colectivo IOÉ } \\
\text { (1998), España }\end{array}$ & $\begin{array}{l}18 \text { historias de vida según varias } \\
\text { variables: edad, sexo estado } \\
\text { civil, estatus social, origen de } \\
\text { discapacidad, situación laboral, } \\
\text { hábitat, etc. }\end{array}$ & $\begin{array}{l}\text { Se trata de historias de vida } \\
\text { social. } \\
\text { Se complementa con entrevistas } \\
\text { y grupos de discusión. } \\
\text { Dimensiones de análisis: todas. }\end{array}$ \\
\hline $\begin{array}{l}\text { El impacto de la } \\
\text { crisis económica en } \\
\text { las personas con } \\
\text { discapacidad y sus } \\
\text { familias }\end{array}$ & $\begin{array}{l}\text { Huete et al. } \\
\text { (2009), España }\end{array}$ & $\begin{array}{l}\text { 3 historias a perfiles diferenciados } \\
\text { por género, el tipo de } \\
\text { discapacidad, situación laboral } \\
\text { y convivencia con personas a su } \\
\text { cargo. }\end{array}$ & $\begin{array}{l}\text { Se emplean otras técnicas } \\
\text { cualitativas donde intervienen } \\
\text { otros agentes: grupo de } \\
\text { discusión y entrevistas. } \\
\text { Dimensiones de análisis: todas. }\end{array}$ \\
\hline
\end{tabular}

Fuente: elaboración propia. 
incluyendo las dimensiones económica, formativa, relacional y residencial, ésta última como eje transversal y distinguiendo entre macrourbano, urbano y rural. Las dimensiones laboral, económica, formativa y residencial son elementos centrales en este trabajo.

\subsection{Grupo de discusión}

El grupo de discusión también ha sido empleado para analizar la inserción laboral de las personas con discapacidad, pues que permite captar los discursos de distintos agentes y los consensos y disensos entre los participantes. El cuadro 6 recoge algunas experiencias y sus características.

En el estudio del Colectivo IOÉ (I998) se exploraron los discursos sociolaborales desde una participación directa de las personas con discapacidad, además de las líneas de pensamiento de diversos agentes, tales como padres y madres de jóvenes con discapacidad intelectual mayoritariamente, lo que plantea la dificultad de la comunicación directa o accesibilidad a la discapacidad intelectual, o parientes (mayoría esposas) de personas con discapacidad que perciben pensiones; empresarios y altos cargos de empresas grandes y medianas del ámbito privado y público, explotaciones familiares y pequeñas empresas; agentes de socialización de la discapacidad (educación, sanidad y trabajo social); trabajadores sin discapacidad del sector secundario; trabajadores con discapacidad del sector secundario y primario, y desde los tres enclaves geográficos (macrourbano, urbano y rural). Este macro estudio se sustenta en un amplio equipo técnico entre el propio grupo de investigadores, las personas con discapacidad y distintos expertos. Como ya se ha comentado anteriormente, este estudio contempla todas las dimensiones citadas a nivel teórico.

El otro macroestudio multicualitativo promovido por el CERMI acerca del impacto de la crisis económica realiza el diseño muestral desde una perspectiva multidimensional, una segregación de los discursos según el género y en comparación con la población activa sin discapacidad, disponiendo de un amplio equipo técnico o colaborador. Del mismo modo, aborda todas las dimensiones de análisis que se han mencionado con anterioridad.

\subsection{Observación participante}

La técnica de la observación participante no presenta una aplicación frecuente aunque tiene el gran potencial de obtener una amplia información. Aporta la riqueza de recoger los diálogos, las actitudes, las emociones, las interacciones y el entorno en tiempo real, permitiendo minimizar las limitaciones comunicativas en el trabajo de campo,

Cuadro 6. Aplicación del grupo de discusión al estudio de inserción sociolaboral de personas con discapacidad

\begin{tabular}{|l|l|l|l|}
\hline Título & $\begin{array}{l}\text { Autoria y } \\
\text { contexto }\end{array}$ & Muestra seleccionada & Aportaciones / Valoraciones \\
\hline $\begin{array}{l}\text { Discapacidad y Trabajo } \\
\text { en España }\end{array}$ & $\begin{array}{l}\text { Colectivo IOE } \\
\text { (1998), Epaña }\end{array}$ & $\begin{array}{l}17 \text { grupos con distintos } \\
\text { participantes: personas con } \\
\text { discapacidad en distintos } \\
\text { contextos, empresarios, } \\
\text { familias, expertos, etc }\end{array}$ & $\begin{array}{l}\text { Se complementa con } \\
\text { entrevistas e historias de vida. } \\
\text { Dimensiones de análisis: todas. }\end{array}$ \\
\hline $\begin{array}{l}\text { El impacto de la } \\
\text { lasis económica en } \\
\text { las personas con } \\
\text { discapacidad y sus } \\
\text { familias }\end{array}$ & $\begin{array}{l}\text { Huete et al. } \\
\text { (2009), España }\end{array}$ & $\begin{array}{l}\text { 3 grupos de discusión: dos con } \\
\text { personas con discapacidad } \\
\text { que trabajan (uno con mujeres } \\
\text { y otro con hombres) y uno con } \\
\text { profesionales. }\end{array}$ & $\begin{array}{l}\text { Sualitativas donde intervienen } \\
\text { otron agentes: historias de vida } \\
\text { y entrevistas. } \\
\text { Dimensiones de análisis: todas. }\end{array}$ \\
\hline
\end{tabular}

Fuente: elaboración propia. 


\begin{tabular}{|c|c|c|c|}
\hline Título & $\begin{array}{l}\text { Autor y } \\
\text { contexto }\end{array}$ & Muestra seleccionada & Aportaciones/Valoraciones \\
\hline $\begin{array}{l}\text { Movilidad } \\
\text { geográfica y } \\
\text { accesibilidad en } \\
\text { las rutas laborales }\end{array}$ & $\begin{array}{l}\text { Velasco et } \\
\text { al. (2014). } \\
\text { España }\end{array}$ & $\begin{array}{l}8 \text { casos sobre los que se realizó } \\
\text { observación participante en el } \\
\text { desplazamiento desde el hogar } \\
\text { hasta el lugar de trabajo. }\end{array}$ & $\begin{array}{l}\text { La observación participante apenas se ha } \\
\text { aplicado al estudio de la discapacidad. } \\
\text { Aporta una amplia información } \\
\text { Dimensiones de análisis: laboral, de salud } \\
\text { y residencial. }\end{array}$ \\
\hline
\end{tabular}

Fuente: elaboración propia.

principalmente de las discapacidades auditivas, intelectuales y mentales.

El estudio de Velasco et al. (20I4) sobre la movilidad y accesibilidad de las rutas de trabajo en la población con discapacidad emplea entre otras técnicas la observación participante sobre 8 casos. Los perfiles se seleccionaron en base al sexo, al tipo de discapacidad, al medio de transporte y al ámbito de población, como puede observarse en el cuadro 7 .

Este estudio es un ejemplo más de la amplitud de la investigación y de la metodología multicualitativa, sustentada por un gran equipo de trabajo y promovido por las entidades representativas de la discapacidad. Aunque en este trabajo la multidimensionalidad analiza directamente la dimensión laboral y residencial (hábitat y acceso al lugar de trabajo), también se aproxima a la dimensión relacional y a la de salud implícitamente.

\section{Conclusiones}

Las metodologías de investigación social presentan limitaciones y potencialidades para el estudio de la discapacidad. Como se ha visto, en la actualidad, a pesar de haber superado la carencia de datos cuantitativos sobre esta temática considerando la dimensión social de la discapacidad como elemento generador de la misma, aún queda una gran tarea por avanzar.
De la revisión teórica y empírica de este trabajo se pueden diferenciar dos tipos de decisiones en el diseño de investigaciones sobre discapacidad: unas vinculadas a los marcos teóricos y conceptuales (cuadro 8); y otras más claramente vinculadas con las metodologías (cuadro 9). El cuadro 8 establece las decisiones conceptuales valoradas en los análisis científicos sobre la inserción sociolaboral de las personas con discapacidad.

En relación al análisis conceptual aplicado, es obvio que la discapacidad es una cuestión de derechos humanos, dando énfasis al Enfoque de Derechos Humanos (EDH), como también se ha podido ver en el desarrollo de este texto. Un factor esencial es la sintonía entre discapacidad y desigualdad social, puesto que tener una discapacidad conlleva una situación de vulnerabilidad respecto a la población sin discapacidad y una asociación directa con la exclusión social. Otro matiz a señalar del análisis es la perspectiva holística del modelo de inserción bio-psico-social en el entendimiento de la naturaleza de la discapacidad y sus barreras sociales, aun reflejando un excesivo centralismo del individuo, así como las reflexiones conceptuales sobre los términos de discapacidad y de diversidad funcional, que no están consensuados actualmente. El análisis del cuadro 8 muestra también la presencia de diversas dimensiones en los estudios sobre esta temática, abordadas de forma explícita como ejes centrales de la metodología o reflejadas implícitamente en relación a las principales variables consideradas. Esta línea argumental avala un enfoque multidimensional 


\begin{tabular}{|c|c|c|}
\hline $\begin{array}{l}\text { Cuadro 8. Decisiones } \\
\text { discapacidad }\end{array}$ & conceptuales de los estudios sobre inserc & sociolaboral de las personas con \\
\hline \begin{tabular}{|l} 
Decisión conceptual \\
\end{tabular} & Opciones / Características & Qué valorar \\
\hline $\begin{array}{l}\text { 1. Concepto } \\
\text { discapacidad }\end{array}$ & $\begin{array}{l}\text { - Invalidez, incapacidad, minusvalía, } \\
\text { diversidad funcional (CIDDM). } \\
\text { - Discapacidad, diversidad funcional (CIF). }\end{array}$ & $\begin{array}{l}\text { Adecuación a la situación social } \\
\text { actual: derechos de ciudadanía. }\end{array}$ \\
\hline $\begin{array}{l}\text { 2. Modelo social } \\
\text { de discapacidad } \\
\text { contemplado }\end{array}$ & $\begin{array}{l}\text { - Médico biológico. } \\
\text { - Modelo social. } \\
\text { - Modelo bio-psico-social. } \\
\text { - Modelo político activista. } \\
\text { - Modelo universal. }\end{array}$ & $\begin{array}{l}\text { Adecuación para un conocimiento } \\
\text { holístico de la inserción sociolaboral. }\end{array}$ \\
\hline $\begin{array}{l}\text { 3. Tipos de } \\
\text { discapacidad } \\
\text { contemplados }\end{array}$ & $\begin{array}{l}\text { - General. } \\
\text { - Tipología básica: física, mental, } \\
\text { intelectual y sensorial. } \\
\text { - Tipología extensa, diferenciando las } \\
\text { subtipologías. }\end{array}$ & $\begin{array}{l}\text { Cobertura y decisiones muestrales. } \\
\text { Necesidad de adaptación de algunas } \\
\text { técnicas a tipología concreta. }\end{array}$ \\
\hline $\begin{array}{l}\text { 4. Dimensiones de } \\
\text { análisis consideradas }\end{array}$ & $\begin{array}{l}\text { Además del trabajo: ingresos, red } \\
\text { sociofamiliar, participación, salud, educación } \\
\text { y contexto residencial. }\end{array}$ & $\begin{array}{l}\text { La vinculación entre las distintas } \\
\text { dimensiones contempladas: (explícitas } \\
\text { o indirectamente) y la vinculación entre } \\
\text { ellas. }\end{array}$ \\
\hline
\end{tabular}

Fuente: elaboración propia.

Cuadro 9. Decisiones metodológicas de los estudios sobre inserción sociolaboral de las personas con discapacidad

\begin{tabular}{|c|c|c|}
\hline Decisión metodológica & Opciones / Características & Qué valorar \\
\hline $\begin{array}{l}\text { 1. Entidad promotora de } \\
\text { la investigación }\end{array}$ & Pública/Privada/Mixta & $\begin{array}{l}\text { Financiación, agentes que } \\
\text { promueven y el papel de éstos. }\end{array}$ \\
\hline $\begin{array}{l}\text { 2. Disciplinas que } \\
\text { realizan la investigación }\end{array}$ & $\begin{array}{l}\text { Medicina, psicología, sociología, educación, } \\
\text { trabajo social... }\end{array}$ & $\begin{array}{l}\text { Qué aporta cada una de ellas. } \\
\text { Disciplina que coordina. }\end{array}$ \\
\hline $\begin{array}{l}\text { 3. Agentes implicados } \\
\text { en la investigación }\end{array}$ & $\begin{array}{l}\text { Familia, profesionales de la discapacidad, } \\
\text { expertos-investigadores, agentes sociales } \\
\text { (empresas, sindicatos). }\end{array}$ & Qué aporta cada agente. \\
\hline $\begin{array}{l}\text { 4. Rol de las personas } \\
\text { con discapacidad en la } \\
\text { investigación }\end{array}$ & $\begin{array}{l}\text { - Co-investigador: ellos mismos investigan. } \\
\text { - Participativo: en todas fases de la } \\
\text { investigación se cuenta con ellos. } \\
\text { - Activo: en una o más fases, aunque no en } \\
\text { todas. } \\
\text { - Pasivo: en ninguna, se investiga a } \\
\text { terceros (cuidadores, expertos...). } \\
\end{array}$ & $\begin{array}{l}\text { Repercusiones del rol sujeto y/u } \\
\text { objeto del estudio. }\end{array}$ \\
\hline $\begin{array}{l}\text { 5. Paradigma de } \\
\text { investigación }\end{array}$ & $\begin{array}{l}\text { - Positivista. } \\
\text { - Interpretativo. } \\
\text { - Emancipador. }\end{array}$ & $\begin{array}{l}\text { Adecuación al objetivo del estudio. } \\
\text { Limitaciones de cada paradigma. }\end{array}$ \\
\hline 6. Técnicas & - Cuantitativa. & $\begin{array}{l}\text { Recursos económicos, fuente } \\
\text { primaria y secundaria y } \\
\text { combinación. }\end{array}$ \\
\hline $\begin{array}{l}\text { 7. Tipo de resultados/ } \\
\text { conclusiones }\end{array}$ & $\begin{array}{l}\text { - } \text { Descriptivos. } \\
\text { - } \text { Críticos. } \\
\text { - } \text { Intervencivos. } \\
\end{array}$ & $\begin{array}{l}\text { Posiciones ideológicas, debilidades } \\
\text { y propuestas. }\end{array}$ \\
\hline 8. Ámbito territorial & Europeo, nacional, regional, local. & $\begin{array}{l}\text { Macro: diferenciación territorial. } \\
\text { Micro: mayor conocimiento local. }\end{array}$ \\
\hline
\end{tabular}

Fuente: elaboración propia. 
en la investigación social sobre el binomio discapacidad/procesos de inserción sociolaboral.

Por su parte, el cuadro 9 refleja las decisiones metodológicas de los estudios analizados. Son diversos los aspectos que engloban este cuadro sinóptico y, posiblemente, se podría ahondar más en ellos. Sin embargo, se considera que es necesario realizar un esfuerzo de síntesis en aras de una mejora aplicativa. La institución o, en algunas ocasiones, las instituciones promotoras de los estudios establecen la procedencia de los recursos económicos y la amplitud de la investigación, existiendo un carácter mayoritariamente público y mixto. De igual modo, éstas condicionan el enfoque de discapacidad del estudio, dirigido hacia una perspectiva general de las discapacidades (una perspectiva conjunta de los cuatro grandes grupos) o hacia a un tipo específico de discapacidad, sin destacar un enfoque sobre otro. La visión general está más identificada con entidades de ámbito nacional e investigadores sociales. Por su parte, el análisis de una discapacidad concreta está más relacionado con la discapacidad que representa dicha entidad y más vinculado con un nivel regional y local.

Los destinatarios y su participación en el trabajo de campo es un contenido relevante en los estudios revisados. El diseño muestral es un elemento básico en todo trabajo de investigación y, en los estudios sobre la inserción laboral de la población con discapacidad, es un valor adicional la participación del propio colectivo aportando una visión directa y, en primera instancia, tiene un doble valor informativo (pensamientos, emociones, aspectos desconocidos, etc.); además, permite el contraste entre lo objetivo y subjetivo sobre la temática tratada. En cuanto a la participación, se resalta la coparticipación del propio colectivo en algunos estudios analizados, reflejándose ésta con la presencia en el equipo investigador de personas con discapacidad y, en otros, es el colectivo quien promueve y desarrolla el estudio.

En relación a los ítems que condicionan las investigaciones, es destacable la importancia del rol que pueden ejercer las personas con discapacidad, al ser éstas las protagonistas y el centro del objeto de estudio, incluso aplicando metodologías de investigación, acción y participación (IAP). Los trabajos que recogen la propia voz del colectivo son escasos; en algunos casos, se recoge la información a través de terceras personas ante las barreras comunicativas con los propios individuos, lo que conlleva a una información parcial.

Dentro de las distintas líneas de investigación se observan diferentes corrientes metodológicas (positivistas, interpretativas o emancipadoras), manifestando un predominio del enfoque sobre los objetivos del estudio, que implica una limitación al conocimiento de la realidad de la población con discapacidad. Se considera necesario realizar estudios con carácter mixto que combinen los distintos paradigmas, más allá del discurso dominante o de la corriente subyacente.

Respecto a las técnicas empleadas destaca un predominio cualitativo, una aplicación cuantitativa desde fuentes secundarias y una complementariedad en el uso combinado de varias técnicas. Las fuentes estadísticas presentan limitaciones por falta de homogeneidad, de regularidad y por un alcance reducido sobre la situación de discapacidad, reflejando solamente una realidad parcial del colectivo. En esta disyuntiva, la combinación de diversas técnicas ofrece una complementariedad al análisis, tanto el uso de cuantitativas y cualitativas como la combinación de diferentes técnicas cualitativas, valorando la relevancia de la información obtenida en cada una de ellas.

Otro aspecto a considerar es la perspectiva de análisis, macrosociológica o microsociológica, en los estudios sobre discapacidad. En el presente trabajo se observa que los estudios sobre el conjunto de las discapacidades así como los análisis cuantitativos de macroencuestas nacionales e internacionales muestran un enfoque macrosociológico. Cabe mencionar que las operaciones estadísticas nacionales, aunque recogen algunos datos regionales y locales, presentan un enfoque nacional o 
internacional donde no todos los datos están desglosados por autonomías y municipalidades. Por su parte, los estudios específicos sobre una determinada discapacidad o los centrados en una entidad concreta, constituyen una visión microsociológica de la discapacidad.

Del análisis de los estudios sobre discapacidad revisados se destacan las ventajas de aplicar metodologías mixta y con enfoque multidimensional. La combinación de distintas técnicas puede aporta una complementariedad y profundidad al análisis del fenómeno social de la discapacidad, ofreciendo un mejor conocimiento de la diversidad y complejidad del fenómeno, así como de su construcción social, que aún está inconclusa.

En la producción empírica sobre la discapacidad es aconsejable tener en cuenta ciertas consideraciones y fundamentos básicos sobre esta temática, con el objetivo de aportar una adecuada construcción científica. En líneas generales, se hace preciso realizar diseños muestrales más exhaustivos en relación con las distintas tipologías de la discapacidad, que garanticen una representatividad más fiable de esta heterogénea población y no sea reducida al mismo grupo de individuos más disponibles, debido a su menor dificultad comunicativa y de movilidad (Toboso-Martín y RogeroGarcía, 20I2). De igual modo, es necesario adaptar las técnicas de investigación social a las características particulares de la persona, garantizando la accesibilidad y favoreciendo la participación del propio colectivo en las investigaciones. Todo ello, con el objetivo de garantizar la accesibilidad universal (IMSERSO, 2004).
Desde el punto de vista teórico, el enfoque multidimensional presenta la realidad social de este colectivo desde la suma de las distintas dimensiones sociales y permite establecer las interrelaciones de los ámbitos básicos para su integración social e identificar los posibles factores de inclusión/exclusión, en correspondencia a cada dimensión.

Estas pautas metodológicas y teóricas, además de ahondar en el conocimiento de la heterogeneidad y complejidad de este fenómeno social, favorecen la adquisición de una concienciación universal e inclusiva de la discapacidad desde el mismo inicio de la labor empírica. Asimismo, se hace preciso ahondar en el debate teórico-conceptual que contribuya al equilibro entre el nivel individual y los factores sociales, donde el modelo universal puede ser una línea de reflexión y avance al modelo biopsicosocial. Según el posicionamiento teórico de partida y el modelo social sobre la discapacidad considerado, los resultados obtenidos sobre la integración sociolaboral de este colectivo pueden ser distintos. En definitiva, el fenómeno de la discapacidad tiene un marcado carácter social que obliga a la ciencia sociológica a contribuir en el entendimiento y desarrollo de esta cuestión. La implicación y la producción empírica y literaria en materia de discapacidad se han incrementado en los últimos años, si bien aún se carece de una sociología de la discapacidad. En este sentido, es importante resaltar el valor y el potencial del enfoque multidisciplinar, incluso de las ciencias en general, que contribuiría a una mayor extensión y calidad formativa sobre esta materia, a unas políticas y acciones más efectivas en la inserción sociolaboral de las personas con discapacidad y, en última instancia, a la integración social. 
Alcover, C.M. y Pérez, V. (2010): Integración de personas con discapacidad en el mercado de trabajo ordinario. Aspectos psicosociales, Madrid: Fundación Adecco.

Almeida, M.E. et al. (2010): "Nuevas retóricas para viejas prácticas. Repensando la idea de diversidad y su uso en la comprensión y abordaje de la discapacidad". Política y Sociedad, 47(I): 27-44.

Anaut, S. y Arza, J. (20I 5): “La exclusión social en los hogares con algún miembro con discapacidad durante el período de crisis en España”. Revista Española de Discapacidad, 3 (I): 7-28.

Anaut, S. et al. (20I4): "La incidencia de la exclusión social en los hogares con personas con discapacidad”, en Laparra M.: VII Informe FOESSA. Madrid: Fundación FOESSA-Cáritas.

Barton, L. (comp.) (2008): Superar las barreras de la discapacidad, Madrid: Ediciones Morata.

Cano, A., Díaz, E., Ferreira, M.A.V., Rodríguez, S. y Toboso, M. (2015): Discapacidad y politicas públicas. La experiencia real de los jóvenes con discapacidad en España, Madrid: Los libros de la catarata.

Colectivo IOE (2012): Discapacidad e inclusión social. Barcelona: Obra social la Caixa.

Colectivo IOÉ (1998): Discapacidad y trabajo en España. Estudio de los procesos de inclusión y exclusión de las personas con discapacidad, Madrid: Ministerio de Trabajo y Asuntos Sociales.

Colectivo IOÉ (I997): La mejora de las condiciones de trabajo y de vida de las personas con discapacidad. Estudios de caso en España, Madrid: European Foundation.

Colectivo IOÉ (I993): “IAP. Introducción en España”. Documentación Social, 92: 59-69.

Díaz Velázquez, E. (2009): "Reflexiones epistemológicas para una sociología de la discapacidad". Revista sociológica de pensamiento crítico, 3 (2), 85-99. España (en línea). <http://www.intersticios.es/article/ view/45 57/3I77>, accesor 9 de mayo de 2015.

Díaz Velázquez, E. (20I0): “Ciudadanía, identidad y exclusión social de las personas con discapacidad". Política y Sociedad, 47 (I): I I 5I 35 .

Díaz Velázquez, E. (20I I): "Estratificación y desigualdad por motivo de discapacidad". Revista Intersticios, 5(I): I 57-I70.

España. Ley 39/2006, de I4 de diciembre de Promoción de la Autonomía Personal y Atención a las personas en situación de dependencia, Boletín Oficial del Estado, núm. 299, de I 5 de diciembre de 2006, pp. 44I 42 a $44 \mathrm{I} 56$.

España. Real Decreto Legislativo I/2013, de 29 de noviembre, por el que se aprueba el Texto Refundido de la Ley General de derechos de las personas con discapacidad y de su inclusión social. Boletín Oficial del Estado, núm. 289, de 3 de diciembre de 2013, pp. 95635 a 95673 .

Ferrante, C. y Ferreira, M.A. (2010): "El habitus de la discapacidad: la experiencia corporal de la dominación en un contexto económico periférico". Política y Sociedad, 47 (I): 8I-IO4.

Ferreira, M.A (2008): “Una aproximación sociológica a la discapacidad desde el modelo social: apuntes caracteriológicos". Revista española de investigaciones sociológicas, I24: I 4 I-I 74 .

Ferreira, M.A. (20I0): "De la minus-valía a la diversidad funcional: un nuevo marco teórico metodológico". Politica y Sociedad, 47 (I): $45-65$.

Garvía, R. (I997): En el País de los Ciegos: la ONCE desde una perspectiva sociológica, Barcelona: Hacer.

Hernández Pedreño, M. (20I0): "El estudio de la pobreza y la exclusión social. Aproximaciones cuantitativas y cualitativas". 
Revista Interuniversitaria de Formación del Profesorado, 69 (24,3), 25-46.

Huete, A. (2012): La discapacidad en las fuentes estadísticas oficiales. Exámen y propuestas de mejora (II). Análisis formal y de contenido sobre discapacidad en las referencias del Centro de Investigaciones Sociológicas (CIS), Madrid: CINCA.

Huete, A. (20I3): "La exclusión de la población con discapacidad en España. Estudio específico a partir de la Encuesta Social Europea". Revista española de discapaciad, I (2): 7-24.

Huete, A. et al. (2009): El impacto de la crisis económica en las personas con discapacidad y sus familias, Madrid: Comité Español de Representante de Minusválidos, CERMI.

IMSERSO (2004): I Plan Nacional de Accesibilidad, 2004-20I2. Madrid: IMSERSO (en línea). $<$ http://sid.usal.es/idocs/F8/FDO I 26Io/pndaa. pdf $>$, acceso I 5 de junio de 20 II.

INE (1999): Metodología. Encuesta sobre discapacidades, deficiencias y estado de salud. España (en línea). http://www.ine.es/prodyser/ pubweb/discapa/disca99meto.pdf, acceso 3 de noviembre de 2015 .

INE (2003): Encuesta de Población Activa. Módulo de personas con discapacidades y su relación con el empleo. Segundo trimestre de 2002. España (en línea). http://www.ine.es/daco/ daco43/metodorep2002.pdf, acceso 3 de noviembre de 2015 .

INE (2008): Encuesta sobre discapacidad, autonomía personal y situaciones de dependencia (EDAD). Metodología. España (en línea). http://www.ine.es/metodologia/t I $5 /$ tI 5304I 8.pdf, acceso 3 de noviembre de 2015.

INE (20I2): Encuesta de Población Activa. Módulo sobre problemas de salud y su relación con el empleo. España (en línea). <http://www.ine. es/daco/daco42/daco42I I/metomodu I I.pdf >, acceso 3 de novimebre de 2015 .

INE (2013): El salario de las personas con discapacidad. Explotación de la Encuesta de Población Activa y de la Base Estatal de Personas con Discapacidad. Metodología y descripción general de la operación. España (en línea). http://www.ine.es/metodologia/t22/ t223033I.pdf, acceso 28 de octubre de 2015.

INE (20I2): Encuesta de integración social y salud. EISS-20 I 2. Metodología. España (en línea). http://www.ine.es/metodologia/tI 5/tI 5304 I 70 I 2. pdf, acceso 28 de octubre de 2015 .

INE (2OI4): El empleo de las personas con discapacidad. Explotación de la Encuesta de Población Activa y de la Base Estatal de Personas con Discapacidad. Metodología y descripción general de la operación. España (en línea). http://www.ine.es/metodologia/t22/ t2230320.pdf, acceso 28 de octubre de 2015 .

Jiménez Lara, A. (2007): “Conceptos y tipologías de la discapacidad. Documentos y normativas de clasificación más relevantes", en R. de Lorenzo García y L.C. Pérez Bueno (coords): Tratado sobre discapacidad. Madrid: ThomsonAranzadi.

Minguijón, J., Penacho, A. y Pac, D. (2005): Discapacidad y empleo, Zaragoza: Instituto Aragonés de Empleo.

Observatorio sobre discapacidad y mercado de trabajo en España (20I4): Informe cero. La situación de las personas con discapacidad en el mercado laboral, Madrid: ODISMET.

OMS (1980): International Classification of Impairments, Disabilities and Handicaps. Ginebra: OMS.

OMS (200I): The International Classification of Functioning, Disability and Health. Luxemburgo (en línea). <http://www.who.int/ classifications/icf/en/\#>, acceso I I de octubre de 2015 .

Organización de las Naciones Unidas, ONU (2006). Convención sobre los Derechos de las Personas con Discapacidad, New York: ONU.

Palacios, A y Romañach, J. (2006): El modelo de la diversidad. La bioética y los derechos humanos como herramientas para alcanzar la plena dignidad en la diversidad funcional, España: Diversitas - AIES.

Pujadas Múñoz, J.J. (I 992): El método biográfico: el uso de las historias de vida en ciencias 
sociales. Madrid: Centro de Investigaciones Sociológicas.

Raya, E., Caparrós, N. y Peña, G.B. (20I2): Atención social a personas con discapacidad. Hacia un enfoque de Derechos Humanos, Buenos Aires: Lumen-Humanitas.

Sanmartín, J. y Serrano, J.M. (I998): Historia antigua del Próximo Oriente. Mesopotamia y Egipto, Madrid: Akal.

Servicio Público de Empleo Estatal, SEPE (2OI 5 ): Informe Anual del Mercado de Trabajo de las Personas con Discapacidad Estatal. España (en línea). http://www.sepe.es/indiceObservatorio/ buscar.do indice $=\mathrm{I} \&$ tipo $=2 \&$ periodo $=$ anual $\& \mathrm{a}$ mbito $=$ Nacional\&tema $=\mathrm{O}_{3}$ \& idioma $=\mathrm{es}$, acceso 5 de noviembre de 2015 .

Toboso-Martín, M. y Rogero-García, J. (20I 2): "Diseño para todos en la investigación social sobre personas con discapacidad". Revista española de investigaciones sociológicas, I40: I63-I72.

Velasco Gisbert, M.L. (20I4): La movilidad geográfica de las personas con discapacidad y la accesibilidad de las rutas de trabajo, Madrid: Red2Red Consultores.

Valles, M.S. (1997): Técnicas cualitativas de investigación social. Reflexiones metodológicas y práctica profesional, Madrid: Síntesis. 